\title{
Below-band-gap waveguiding behaviors of a weakly index-guided GaAs/AlGaAs quantum well laser
}

\author{
Horng-Shyang Chen, Shun-Lee Liu, C.C. Yang*, Yean-Woei Kiang \\ Department of Electrical Engineering, Graduate Institute of Electro-Optical Engineering, \\ and Graduate Institute of Electronics Engineering, National Taiwan University, 1, Roosevelt Road, Sec. 4, Taipei, Taiwan, ROC
}

Received 2 May 2002; received in revised form 2 September 2002; accepted 10 March 2003

\begin{abstract}
We report the reduced waveguiding efficiency for the signals around $1560 \mathrm{~nm}$ as the injection current of an GaAs/ AlGaAs multiple quantum well laser diode (lasing wavelength at $840 \mathrm{~nm}$ ) with a ridge-loading waveguide configuration increased. This reduction trend stopped when the injection current reached the threshold condition of the laser diode. The decreased waveguide transmission and the more expanded mode profile indicated the variation of the effective refractive index gradient in the lateral dimension with injection current. This variation was due to the refractive index decrease with increasing carrier density even below band gap. A slab waveguide model was used to simulate the lateral mode profile variation with injection current. The refractive index differences between the guiding layer and claddings in the slab waveguide model provided estimates of refractive index contrasts of the laser diode at a concerned wavelength under various injection conditions.
\end{abstract}

(c) 2003 Elsevier Science B.V. All rights reserved.

\section{Introduction}

Waveguides are key structures for various optoelectronics devices. The confinement factor and attenuation constant of an optoelectronics waveguide can influence the operation efficiency of such a device [1-3]. For instance, the waveguiding efficiency or confinement factor of a semiconductor laser or optical amplifier is an important factor for improving the gain constant. Waveguiding be-

\footnotetext{
${ }^{*}$ Corresponding author. Tel.: +886-2-2365-7624; fax: +8862-2365-2637.

E-mail address: ccy@cc.ee.ntu.edu.tw (C.C. Yang).
}

haviors of such an active device are strongly related to its active region carrier density, which significantly controls its refractive index variation. It is usually believed that the refractive index decreases with increasing carrier density [4-7]. This trend leads to the positive value of the linewidth enhancement factor [8]. Because the refractive index decreases monotonically from a local maximum near the resonance wavelength as wavelength increases, unless other resonance processes occur [4]. It is natural to expect a lower material refractive index below the band gap when a larger current is injected onto the material.

In a ridge-loading laser diode, the injection of current onto the top of the ridge is expected to 
diffuse into the regions outside the ridge-loading area [9]. Based on the refractive index dependence on carrier density described above, such a carrier diffusion process may result in waveguide index profile variation and hence waveguiding property changes. Such changes may occur for wavelengths near and below band gap.

In this paper, we report the observation of decreasing waveguiding efficiency with increasing injection current below band gap in a GaAs/ AlGaAs multiple quantum well laser of a ridgeloading waveguide configuration. At $1560 \mathrm{~nm}$ in wavelength, the waveguiding efficiency in the lateral dimension was reduced as the injection current increased. The reduction of waveguiding efficiency saturated when the threshold condition was reached. The variation of waveguiding efficiency is attributed to the lateral index profile alteration at carrier density change. A slab waveguide model is assumed for estimating the refractive index gradient variation with injection current. This paper is organized as follows: in Section 2, the specifications of the fabricated laser diode are described and the experimental procedures are presented. Then, the characteristics of mode profiles and waveguiding attenuation of below-band-gap optical signals are reported in Section 3. Here, their variations with injection current are interpreted. In Section 4 , the slab waveguide model is used for estimating the refractive index distribution variations. Finally, conclusions are drawn in Section 5.

\section{Laser diode fabrication and experimental proce- dures}

The fabrication of the device is based on a GaAs/AlGaAs quantum well structure. Between the $1.5-\mu \mathrm{m}$ p- and n-type $\mathrm{Al}_{0.4} \mathrm{Ga}_{0.6}$ As cladding, the five $\mathrm{GaAs} / \mathrm{Al}_{0.25} \mathrm{Ga}_{0.75} \mathrm{As}$ quantum wells, with $6.5 \mathrm{~nm}$ well width and $20 \mathrm{~nm}$ barrier width, were sandwiched by the $100 \mathrm{~nm} i$ - $\mathrm{Al}_{0.25} \mathrm{Ga}_{0.75} \mathrm{As}$ separate confinement layer on each side. Laser diodes were fabricated with ridge-loading waveguides of 4 $\mu \mathrm{m}$ in ridge width and $1-\mu \mathrm{m}$ etching depth on the $1.5-\mu \mathrm{m}$ thick p-type cladding layer. The device length was $500 \mu \mathrm{m}$. Current was injected from the top of the ridge of a fabricated laser diode. Near- field mode profiles of the laser waveguide was monitored with an out-coupling objective $(40 \times)$ and an IR camera. Fig. 1 shows the $L-I$ curve of a fabricated laser diode. As clearly seen that the threshold injection current is about $75 \mathrm{~mA}$.

As shown in Fig. 2, we end-fire coupled a cw laser at $1560 \mathrm{~nm}$ of very narrow linewidth, which was from an external-cavity, tunable semiconductor, into the fabricated $\mathrm{GaAs} / \mathrm{AlGaAs}$ laser diode. The half-wave plate and polarizer before the laser device were used for controlling input power and polarization. The output light near $840 \mathrm{~nm}$ from the laser diode was removed with a low-pass color filter when the transmitted $1560 \mathrm{~nm}$ signal was to be monitored. With this filter, no signal could be detected if we turned off the $1560 \mathrm{~nm}$ laser. Also, an aperture was used for clipping the stray light, which

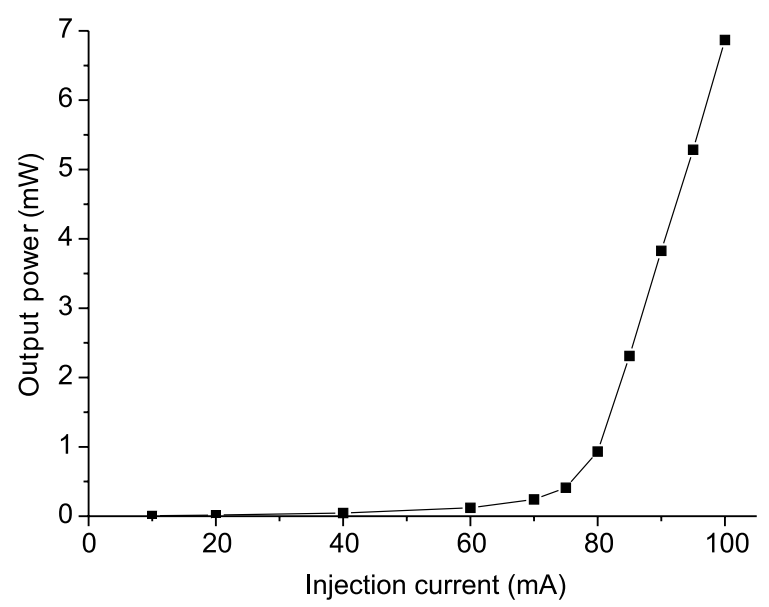

Fig. 1. $L-I$ curve of the fabricated GaAs/AlGaAs quantum well laser diode. The threshold current is about $75 \mathrm{~mA}$.

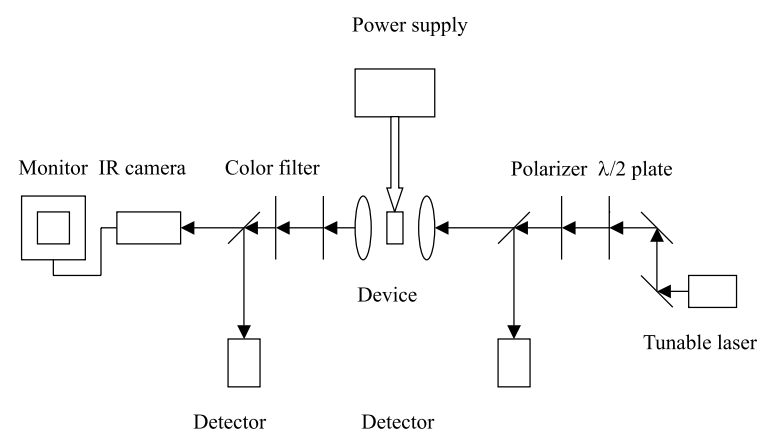

Fig. 2. Experimental setup of signal transmission measurements. 
did not transmit along the waveguide. The output mode profiles were monitored with the IR camera.

\section{Waveguiding characteristics of below-band-gap signals}

With the input power at $2.46 \mathrm{~mW}$ (the end-fire coupling efficiency is estimated to be lower than $1 \%$ ), the four parts of Fig. 3 show the near-field mode patterns of $1560 \mathrm{~nm}$ signal transmission with 0 (a), 40 (b), 60 (c) and 100 (d) $\mathrm{mA}$ injection currents. From Fig. 3, one can see the increasing trend of mode size as the injection current increases. This trend can be better understood with the lateral line scan results in Fig. 4 and the full-width-at-halfmaximum (FWHM) results in Fig. 5. In Figs. 4 and 5, parts (a) and (b) correspond to the results in the lateral and vertical dimensions, respectively. Note that because the near-field mode intensity profiles have been magnified and imaged onto the IR camera, it is difficult to trace their accurate scales. The scales with arbitrary unit (a.u.) are given in Figs. 3-5. From Figs. 4 and 5, one can clearly see that the mode size in the lateral dimension increases more significantly with increasing injection current although that in the vertical direction also shows an increasing trend.

Fig. 6 shows the variations of 1560 -nm signal transmission power of the (a) TE and (b) TM polarization with injection current at various input power levels. In either TE or TM case, output power decreases with injection current. The output power level saturates when the injection current is larger than $75 \mathrm{~mA}$ (threshold condition). The saturation trend indicates that the variations of waveguiding behaviors are strongly related to carrier density in the device because the carrier density in the active region becomes clamped when a laser reaches the threshold condition.

From Figs. 3-6, one can see the decreasing guiding efficiency of the laser diode waveguide for the signal at about half the band gap as the injection current increases. The lower transmission in the situation of a higher injection current can be due to the process of free carrier absorption of 1560 $\mathrm{nm}$ signal. However, the changed waveguide mode
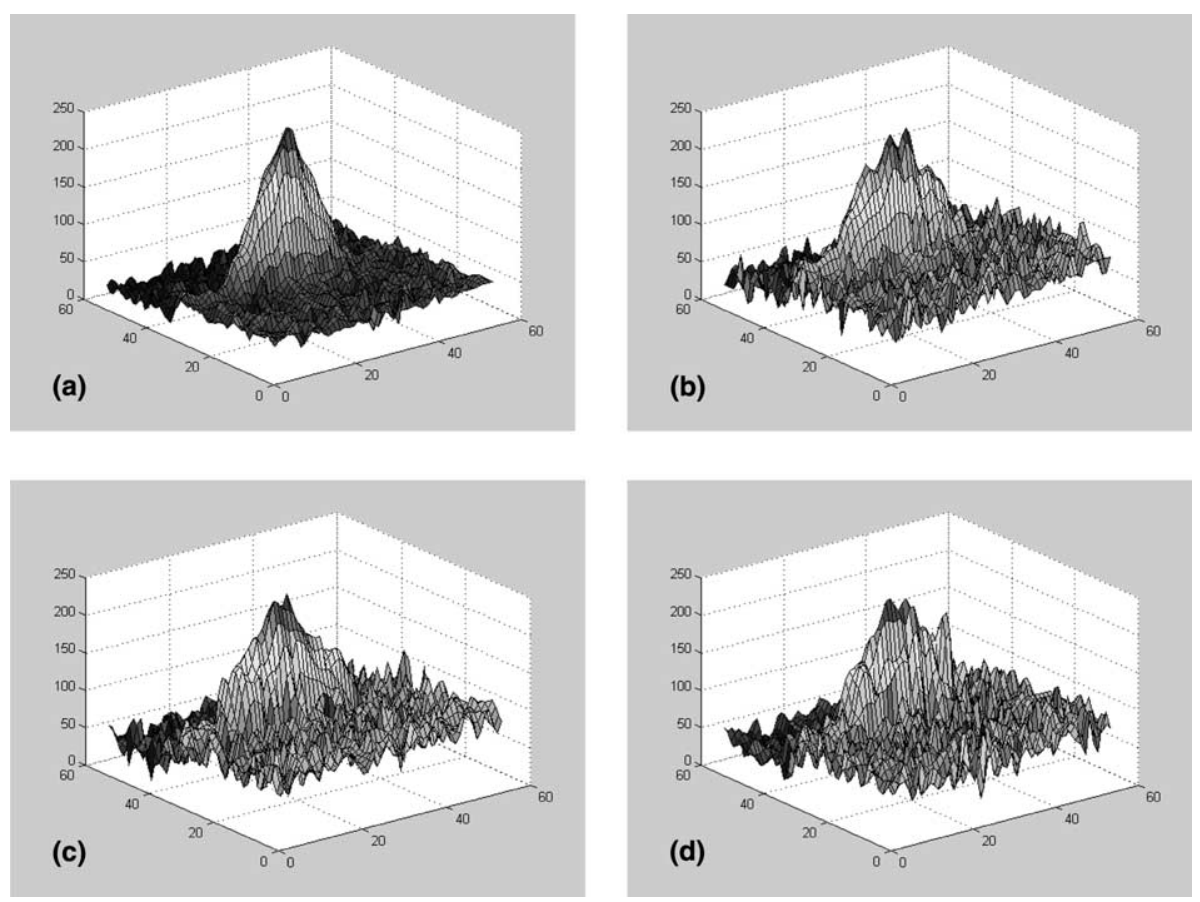

Fig. 3. 3D intensity distributions of the near-field mode patterns of the TE signal at $1560 \mathrm{~nm}$ with (a) 0 , (b) 40 , (c) 60 and (d) $100 \mathrm{~mA}$ injection currents. 

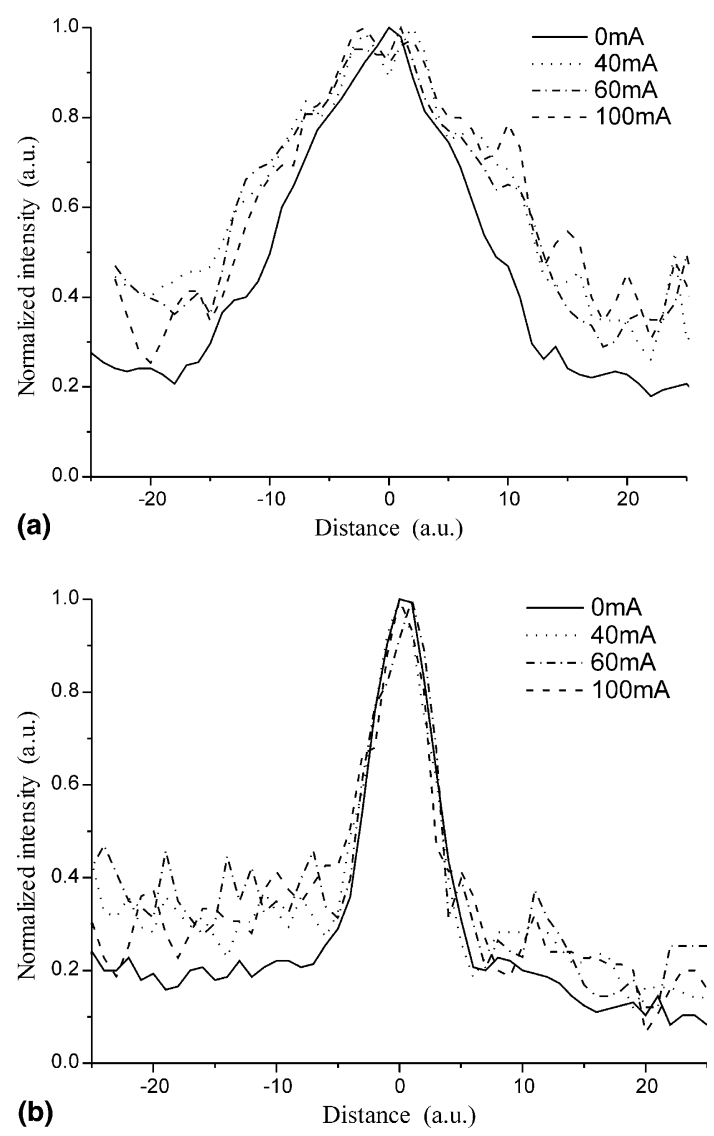

Fig. 4. Line scan intensity distributions from the results in Fig. 3 in the (a) horizontal and (b) vertical directions.

profile reveals that the refractive index distribution, at least in the lateral dimension, is varied as the injection current is increased. This phenomenon can be attributed to the decreased effective refractive index in the regions under the waveguide ridge as carrier density increases (before the threshold condition). Although the effective refractive index outside the ridge region may also be decreased due to carrier diffusion, the gradient of lateral refractive index distribution becomes shallower. In this situation, waveguide mode profile spreads outward and the confinement factor is reduced. Therefore, the more extended evanescent field results in higher waveguide loss due to the perturbations in the waveguide cladding regions. Such a variation in the refractive index distribution would saturate as the carrier density is clamped at the threshold
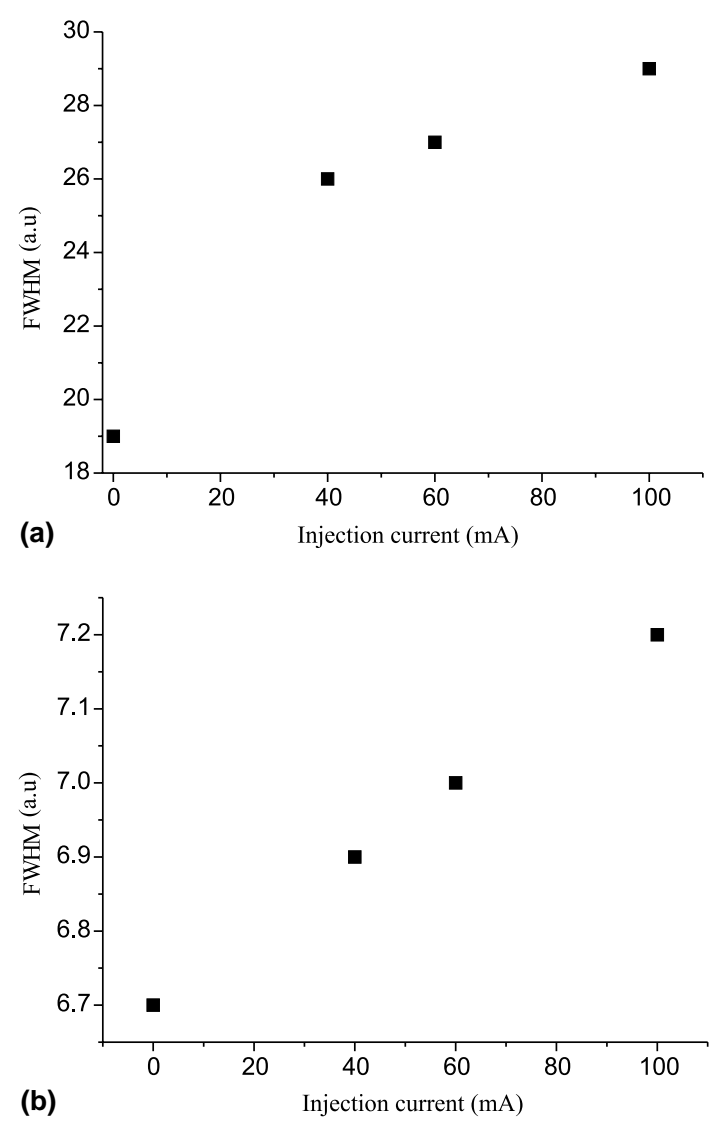

Fig. 5. FWHM of the intensity distributions shown in Fig. 4: (a) horizontal width and (b) vertical width.

condition. From such arguments, one can see that current diffusion may affect less the waveguide mode variation. Although the density of diffused carriers (and hence the refractive index in this region) may increase (decrease) with injection current without clamping at threshold, it seems that waveguide mode is not much influenced by this part of refractive index variation. Here, actually an interesting issue, which deserves further exploration, can be raised: How far in the lateral dimension does carrier density clamping occur at laser threshold outside the ridge-loading regions? Since the variations of wave propagation properties stop at laser threshold, either carrier density clamping occurs in the effective waveguide cladding regions or carrier diffusion does not affect much the refractive index variation in the claddings. 

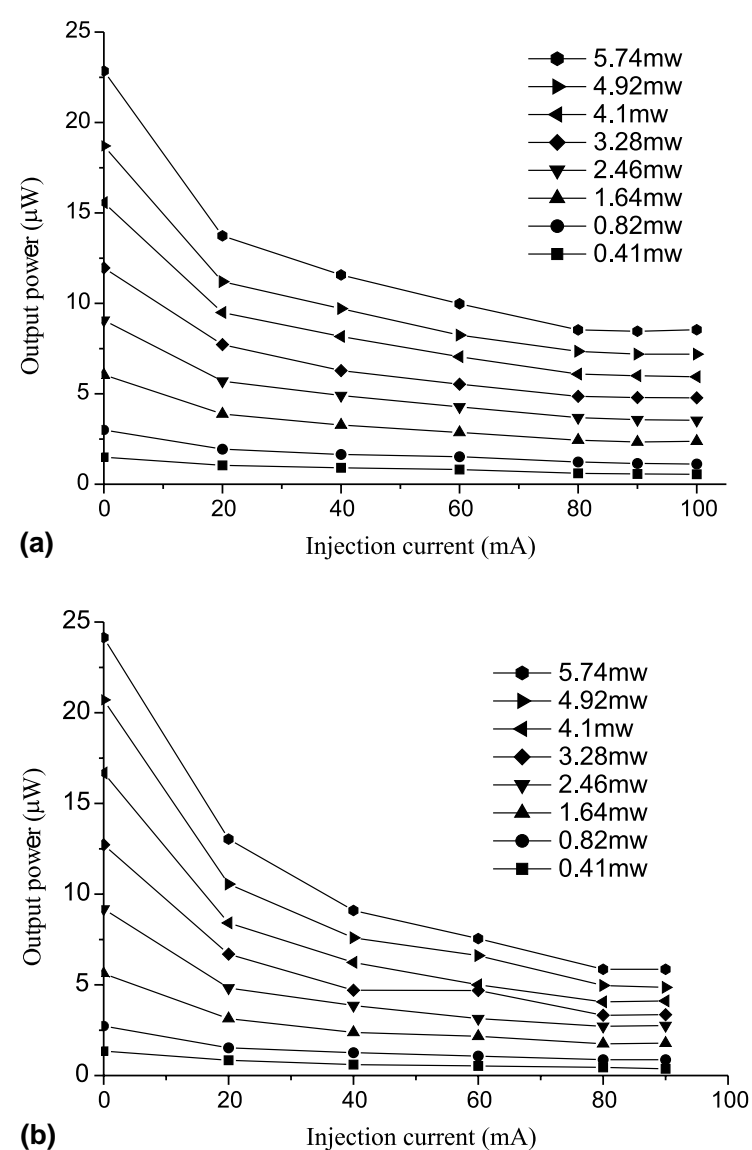

Fig. 6. Output power variations with injection current for the $1560 \mathrm{~nm}$ signal in the (a) TE and (b) TM polarization at several input power levels.

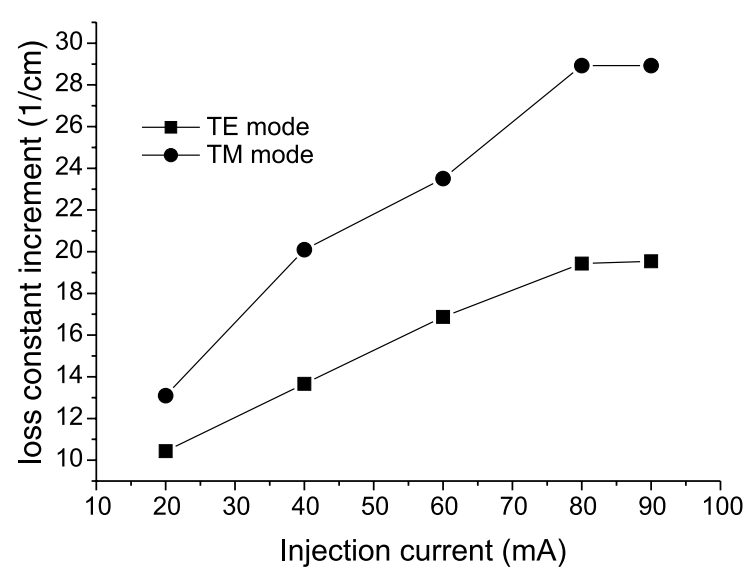

Fig. 7. Variations of the increments of linear loss coefficients with injection current for the TE and TM polarization.
Fig. 7 shows the variation of loss constant increments with injection current in both $\mathrm{TE}$ and TM modes. The loss constant increment means the increased amount of attenuation coefficient compared with the value at zero injection current. One can see that the linear loss coefficients increase by $19 \mathrm{~cm}^{-1}$ in the TE mode and by $29 \mathrm{~cm}^{-1}$ in the TM mode when the threshold condition is reached. The TM mode is more sensitive to current injection.

\section{Estimation of refractive index distribution vari- ations}

To demonstrate the waveguide mode profile variation with the refractive index distribution change, we adopted a slab waveguide model based on the effective index method [10]. In this model, we assumed that the regions below and outside the waveguide ridge formed the guiding layer and claddings of a symmetric slab waveguide. We then calculated the waveguide mode intensity profiles with the appropriately chosen refractive indices of the guiding layer and claddings. Fig. 8 shows four intensity profiles with the refractive index pairs as $\left(n_{\mathrm{f}}, n_{\mathrm{c}}\right)=(3.402,3.3995),(3.4,3.3986),(3.3985$, $3.3978)$ and $(3.3978,3.3974)$, respectively, with increasing profile width. Here, $n_{\mathrm{f}}$ and $n_{\mathrm{c}}$ are the refractive indices of the guiding layer and clad-

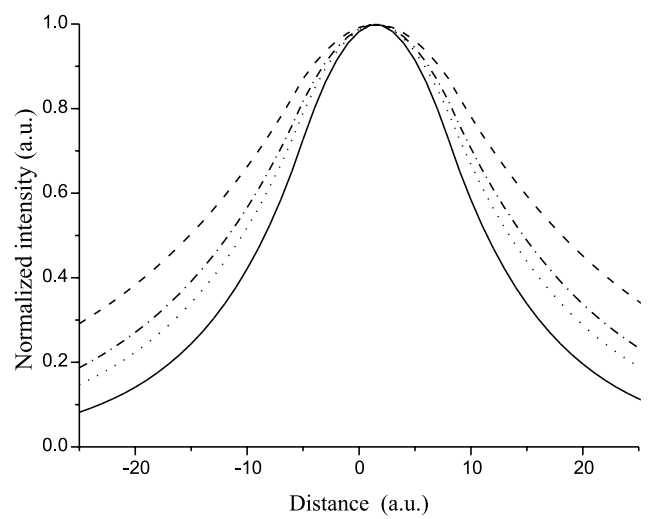

Fig. 8. Lateral intensity distributions based on the slab waveguide model. The four curves with increasing width correspond to the refractive index pairs as $\left(n_{\mathrm{f}}, n_{\mathrm{c}}\right)=(3.402,3.3995)$, $(3.4,3.3986),(3.3985,3.3978)$ and $(3.3978,3.3974)$, respectively. 
dings, respectively. The refractive index values were appropriately chosen such that the FWHMs of the intensity profiles were correspondingly the same as those in Fig. 4(a). Also, single-mode propagation was guaranteed, as observed in experiments. Both refractive indices in the guiding layer and claddings decreased, with that in the guiding layer decreasing faster, as the intensity profile width increased. This decreasing refractive index trend and hence increasing profile width trend correspond to the increase of injection current in Fig. 4(a). However, because the variation of the injection current density in the guiding layer was expected larger than that in the claddings in increasing injection current, the refractive index decrease in the guiding layer was assumed larger. From the refractive index values shown above, one can see that the index difference between the guiding layer and claddings varies from 0.0025 to 0.0004 as the profile width varies from the case of $0-100 \mathrm{~mA}$ injection current. Although such index difference values are not uniquely existent, they should provide estimates of the index contrast variations at the concerned wavelength under various injection conditions. In other words, with the measurements presented in this paper and a suitable waveguide model, we can extract the information of index contrast variation with injection current at a certain wavelength below band gap. If the optical signal wavelength can be closer to the band gap and a material refractive index model can be well adopted, it is feasible to obtain the index contrast variation around the band gap during current injection onto a laser diode.

\section{Conclusions}

In summary, we have reported the decreasing waveguiding efficiency for the signal about one half the band gap as the injection current of an GaAs/AlGaAs multiple quantum well laser diode with a ridge-loading waveguide configuration was increased. The laser operation wavelength was 840 $\mathrm{nm}$. With the signal at $1560 \mathrm{~nm}$, the decreased waveguide transmission and the more expanded mode profile indicated that the effective refractive index distribution in the lateral dimension was varied with injection current. The decreased material refractive indices in both regions under and outside the waveguide ridge resulted in refractive index distributions of weaker guiding. This trend stopped when the laser threshold condition was reached. A slab waveguide model was used to simulate the lateral mode profile variation with injection current. The refractive index differences between the guiding layer and claddings in the slab waveguide model provided estimates of refractive index contrasts of the laser diode at the concerned wavelength under various injection conditions. Such measurement and simulation procedures may provide a means of estimating refractive index contrast variation of a laser diode around the lasing wavelength.

\section{Acknowledgements}

This research was supported by National Science Council, The Republic of China, under the Grants of NSC 90-2112-M-002-052, NSC 90-2215E-002-027, and NSC 90-2215-E-002-041.

\section{References}

[1] R.J. Deri, E. Kapon, IEEE J. Quantum Electron. 27 (1991) 626.

[2] V.R. Chinni, C.R. Menyuk, Y.J. Chen, IEEE Trans. Photon. Techol. Lett. 3 (1991) 1120.

[3] S. Murata, A. Tomita, A. Suzuki, IEEE Photon Technol. Lett. 5 (1993) 16.

[4] B.R. Bennett, R.A. Soref, J.A. Del Alamo, IEEE J. Quantum Electron. 26 (1990) 113.

[5] S. Shin, C.B. Su, IEEE Photon Technol. Lett. 5 (1993) 981.

[6] D.A. Trivedi, N.G. Anderson, IEEE J. Select. Topics Quantum Electron. 2 (1996) 197.

[7] W.L. Li, Y.K. Su, D.H. Jaw, IEEE J. Quantum Electron. 33 (1997) 416.

[8] C.H. Henry, IEEE J. Quantum Electron. QE-18 (1982) 259.

[9] L.A. Coldren, S.W. Corzine, Diode Lasers and Photonic Integrated Circuits, Wiley, New York, 1995.

[10] e.g., H.A. Haus, in: Waves and Fields in Optoelectronics, Section 6.3, Prentice-Hall, Englewood Cliffs, NJ, 1984, p. 163. 